\title{
LONG-TERM SOLAR CYCLE EVOLUTION: REVIEW OF RECENT DEVELOPMENTS
}

\author{
I. G. USOSKIN ${ }^{1}$ and K. MURSULA ${ }^{2}$ \\ ${ }^{1}$ Sodankylä Geophysical Observatory (Oulu unit), POB 3000, FIN-90014, \\ University of Oulu, Finland \\ ${ }^{2}$ Department of Physical Sciences, POB 3000, FIN-90014, University of Oulu, Finland
}

(Received 21 August 2003; accepted 29 September 2003)

\begin{abstract}
The sunspot number series forms the longest directly observed index of solar activity and allows to trace its variations on the time scale of about 400 years since 1610 . This time interval covers a wide range from seemingly vanishing sunspots during the Maunder minimum in 16451700 to the very high activity during the last 50 years. Although the sunspot number series has been studied for more than a century, new interesting features have been found even recently. This paper gives a review of the recent achievements and findings in long-term evolution of solar activity cycles such as determinism and chaos in sunspot cyclicity, cycles during the Maunder minimum, a general behaviour of sunspot activity during a great minimum, the phase catastrophe and the lost cycle in the beginning of the Dalton minimum in 1790s and persistent 22-year cyclicity in sunspot activity. These findings shed new light on the underlying physical processes responsible for sunspot activity and allow a better understanding of such empirical rules as the Gnevyshev-Ohl rule and the Waldmeier relations.
\end{abstract}

\section{Introduction}

In order to study the statistical properties of solar activity one needs some numerical characteristics related to the entire Sun (or its significant part) which reflect its main activity features. Such characteristics are called indices of solar activity. Although there are many different indices such as those based on faculae, flares, coronal holes, and electromagnetic radiation in various bands $(10.7 \mathrm{~cm}$ radio flux, green corona, etc.), the number of sunspots on the solar disk (so called sunspot activity) is the most famous and widely used index of solar activity. It is based on the longest series of continuous solar observations and reflects the varying strength of the hydromagnetic dynamo process which generates the solar magnetic field. Regular sunspot observations were started by Galileo in 1610 soon after the invention of the telescope. Since that time, sunspot observations were more or less regular covering nearly four hundred years by routine observations. Sunspot number series is the most used index of solar activity and probably the most analyzed time series in astrophysics.

The most pronounced feature of solar activity is the 11-year cycle, also called the Schwabe cycle. This cycle dominates the sunspot activity during almost the 
whole observed time interval, but it is far from a simple sinusoidal wave. Instead, it varies in amplitude, period (length) and shape on different time scales.

Although sunspot activity has been studied for a more than century and numerous books and reviews have been published in this area (e.g., Waldmeier, 1961; Vitinsky, 1965; Kuklin, 1976; Vitinsky, Kopecký, and Kuklin, 1986; Wilson, 1994), some new interesting results related to the long-term variation of sunspot activity have appeared during the last few years. This paper aims to review some of these recent findings and suggestions and to provide a brief overview of the long-term solar cycle evolution. Because of the brevity of this paper, we have to leave some relevant topics, such as, e.g., the spatial distribution of sunspot activity (asymmetry of the latitudinal distribution, active longitudes of sunspot formation, etc,) beyond the scope of this review.

\section{Sunspot Activity Time Series}

\subsection{WOLF SUNSPOT NUMBER (WSN) SERIES}

From solar observations one can measure the number of sunspot groups, $G$, and the number of individual sunspots in all groups, $N$, visible on the solar disc. Then the relative sunspot number can be defined as

$$
R_{z}=k(10 G+N),
$$

where $k$ denotes the individual correction factor which compensates differences in observational techniques and instruments used by different observers, and is used to normalize different observations to each other. This $R_{z}$ quantity, called the Wolf or Zürich sunspot number (called WSN henceforth), was introduced by Rudolf Wolf of Zürich Observatory. $R_{z}$ is calculated for each day using only one observation made by the 'primary' observer (judged as the most reliable observer during a given time) for the day. The primary observers were Staudacher (1749-1787), Flaugergues (1788-1825), Schwabe (1826-1847), Wolf (1848-1893), Wolfer (1893-1928), Brunner (1929-1944), Waldmeier (1945-1980), and Koeckelenbergh (since 1980). If observations by the primary observer are not available for a certain day, the secondary, tertiary, etc., observers are used. The hierarchy of observers is given by Waldmeier (1961). The use of only one observer for each for aims to make $R_{z}$ a homogeneous time series. On the other hand, such an approach ignores all other available observations. If no sunspot observations are available for Borne period, the data gap is filled, without notice in the final WSN series, using an interpolation between the available data and employing also some proxy data. There are also some uncertainties in the definition of $G$ and $N$. Depending on the observational conditions (e.g., clouds) some small spots can be missed and the separation of cores in mixed groups and spots may be difficult. These problems were discussed in detail, e.g., by Vitinsky, Kopecký, and Kuklin (1986) who estimated such systematic uncertainties to be about $25 \%$ in monthly sunspot numbers. 


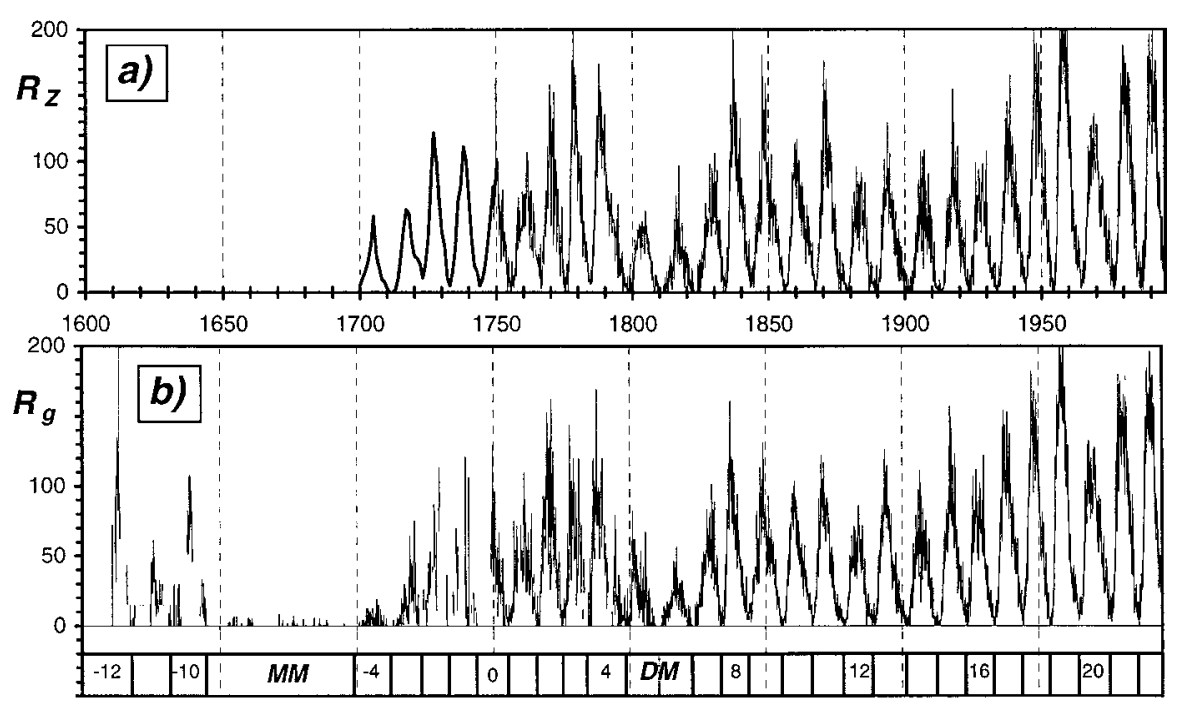

Figure 1. Sunspot activity since 1610. (a) Monthly (since 1749) and yearly (1700-1749) Wolf sunspot number series. (b) Monthly group sunspot number series. Standard (Zürich) cycle numbering as well as the Maunder (MM) and Dalton (DM) minima are shown in the lower panel.

The bulk of the WSN series is based on observations performed at the Zürich Observatory during 1849-1981 using almost the same technique. This part of the series is fairly stable and homogeneous. The official Wolf series starts in 1749 in the middle of solar cycle 0 (see Figure $1(\mathrm{a})$ ). Before 1749, only yearly $R_{z}$ values are available. However, prior to the regular observations at the Zürich Observatory there were many gaps in data during 1749-1849 that were interpolated. Therefore, the WSN series is a combination of direct observations and interpolations for the period before 1849. This results in possible errors and inhomogeneity of the series for those times (see, e.g., Vitinsky, Kopecký, and Kuklin, 1986; Wilson, 1998; Letfus, 1999, and references therein). The quality of the Wolf series before 1749 is rather poor and hardly reliable (Hoyt and Schatten, 1998). Therefore, the WSN series can be analyzed only for the period since 1849 or, with caveats, since 1749 .

\subsection{GROUP SUNSPOT NUMBER (GSN) SERIES}

A new series of sunspot activity called the group sunspot numbers (GSN - see Figure 1(b)) was introduced a few years ago (Hoyt and Schatten, 1998). The daily group sunspot number $R_{g}$ is defined as follows:

$$
R_{g}=\frac{12.08}{n} \sum_{i} k_{i}^{\prime} G_{i}
$$

where $G_{i}$ is the number of sunspot groups recorded by $i$ th observer, $k^{\prime}$ is the observer's individual correction factor, $n$ is the number of observers for the particular 
day, and 12.08 is a normalization number scaling $R_{g}$ to $R_{z}$ values for the period of 1874-1976. $R_{g}$ is more robust than $R_{z}$ since it does not include the number of individual spots. The GSN series includes not only one 'primary' observation but all available observations. This approach allows to estimate systematic uncertainties of the resulting $R_{g}$ values: about $10 \%$ before 1640, less than 5\% in 1640-1728 and in $1800-1849,15-20 \%$ in 1728-1799, and about 1\% since 1849 (see Figure 5 in Hoyt and Schatten, 1998).

The new GSN series includes all available archival records of sunspot observation. The new database compiled by Hoyt and Schatten consists of 455242 observations from 463 observers, about $80 \%$ more daily observations than the WSN series. It has been shown that the GSN series is more reliable and homogeneous than the WSN series before 1849, but the two series closely agree with each other for recent times (Hoyt and Schatten, 1998; Letfus, 1999). Also, the main solar cycle characteristics as obtained from GSN series are similar to WSN series (Hathaway, Wilson, and Reichmann, 2002). The GSN series does not include interpolated data and therefore allows to evaluate the data coverage for each period and to estimate related errors. The GSN series covers the period since 1610 (starting with solar cycle -12), covering thus a 140 years longer period than the WSN series. It is particularly interesting that the period of the Maunder minimum (1645-1715) was surprisingly well covered with daily observations which allows for a detailed analysis of sunspot activity during this great minimum. On the other hand, GSN still contains uncertainties and possible inhomogeneities (see, e.g., Letfus, 2000). However, the great advantage of this series is that these uncertainties can be estimated and taken into account (Usoskin, Mursula, and Kovaltsov, 2003b). The construction of the GSN series and the fact that all basic observations (that are hidden in the WSN series) are included therein, allowing to estimate the uncertainties of the results, were fundamental for the many recent discoveries about long-term sunspot activity.

\subsection{INDIRECT SOLAR PROXIES}

In addition to the regular direct solar observations, there are also indirect solar proxies which are used to study solar activity especially in the pre-telescopic era.

Visual observations of aurorae borealis form a fairly regular series reflecting geomagnetic activity caused by transient phenomena in the varying solar wind (e.g., Silverman, 1983, 1992, 1998; Křivský and Pejml, 1988; Schröder, 1992). Although auroral activity reacts to coronal and interplanetary features rather than the momentary and spatially restricted magnetic fields on the Sun's surface, there is a strong correlation between the long-term occurrence of sunspot numbers and the frequency of auroras. Unfortunately, auroral observations were not done systematically in the early years which makes it difficult to produce a homogeneous data set (see, e.g., Silverman, 1992, 1998). 
Another proxy of solar activity is formed by the data on cosmogenic radionuclides (e.g., ${ }^{10} \mathrm{Be}$ and ${ }^{14} \mathrm{C}$ ) which are produced by cosmic rays in the Earth's atmosphere (e.g., Stuiver and Quay, 1980; Beer, Tobias, and Weiss, 1990). After a complicated transport in the atmosphere they are stored in natural archives such as polar ice, trees, marine sediments, etc. This process is affected also by changes in the geomagnetic field and climate. Cosmic rays experience the heliospheric modulation due to the solar wind and the frozen-in solar magnetic field. The intensity of modulation depends on solar activity and, therefore, the cosmic ray flux and the ensuing cosmogenic isotope intensity depend inversely on solar activity. An important advantage of the cosmogenic data is that they are based upon quantities measured nowadays in laboratories. In contrast to fixed historical archival data (such as sunspot or auroral observations) this approach allows to obtain homogeneous data sets with stable quality and to improve the quality of data with the invention of new methods (such as accelerator mass spectrometry). The cosmogenic isotope data are the only regular indicator of solar activity on the very long-term scale but they can not always resolve details of individual solar cycles.

Some fragmentary data on naked-eye observations of sunspots exist for quite early times, mostly from Oriental sources (see, e.g., Wittmann and Xu, 1987; Yua and Stephenson, 1988). Even though official Chinese chronicles are fairly reliable, these data are not straightforward to interpret and their observational methods are unknown. These data are also seriously contaminated by meteorological or other phenomena. Only about $30 \%$ of Chinese naked-eye sunspot observations were confirmed by direct telescopic data after 1848 (Wittmann and Xu, 1987; Letfus, 2000). Another problem is that the record of naked-eye observations are fragmentary and strongly depend on the frequency of observations. On the other hand, this is a unique set of information of sunspot activity on the long-term scale.

There have been attempts to extend the sunspot series back in time extrapolating the statistical properties of the WSN record (e.g., Nagovitsyn, 1997; De Meyer, 1998; Rigozo et al., 2001). Such models construct, e.g., a modulated carrier frequency or a multi-harmonic representation of the measured SN, which is then extrapolated backward in time. The disadvantage of this approach is that it is not a reconstruction based upon measured or observed quantities but rather a 'prediction' of the SN based on extrapolation. Clearly such models cannot include periods exceeding the time span of observations upon which the extrapolation is based. Hence, the pre- or post-diction becomes increasingly unreliable with growing extrapolation time and its accuracy is hard to estimate. Some models have also tried to unite the extrapolation and the proxy methods. E.g., Schove (1955) fitted the slightly variable but phase-locked carrier frequency, corresponding to the 11year cycle, to fragmentary data from naked-eye sunspot observations or auroral sightings. 


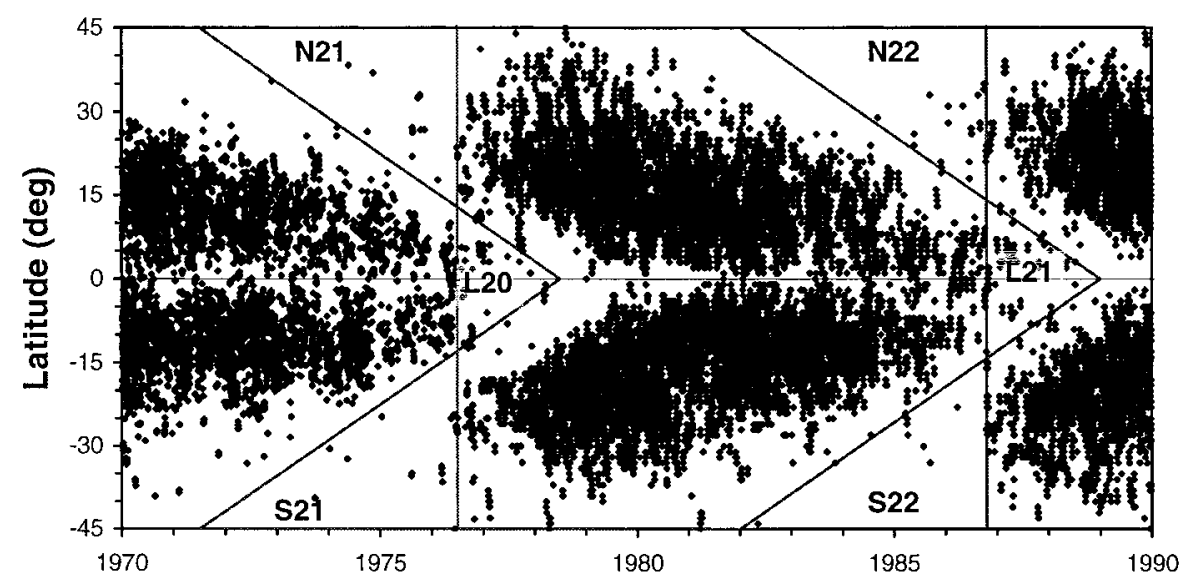

Figure 2. The Maunder butterfly diagram of sunspots for cycles 20-21. Vertical lines denote the times of official minimum. Inclined lines separate the two cycles.

\section{Characteristics of the Solar Cycle}

\subsection{DEFINITION OF CYCLE AND CYCLE LENGTH}

Usually the total number of spots on the solar disk is used to define the sunspot cycle. Since 1874, the location of sunspots on the solar disk was recorded on a routine basis at the Greenwich Solar Observatory. The latitude-time diagram of sunspot occurrence is known as the Maunder butterfly diagram (see Figure 2). Sunspots belonging to the new cycle appear first at higher latitudes and later the activity gradually moves to lower latitudes. This is known as the Spörer law. One can see that, when projected onto the time axis, two successive cycles overlap during a few years around the sunspot minimum. Sunspots of one cycle exist during quite a long period of about $15-17$ years.

Strictly speaking, one should separate the sunspots of the 'old' and 'new' cycle sunspots (between inclined lines in Figure 2), thus taking the overlap into account. Accordingly, some scientists (see, e.g., Pelt et al., 2000; Li et al., 2002) have studied these extended cycles. However, the common practice of separating cycles by the minimum sunspot number times (vertical lines) is often a reasonable approximation and the only possibility for times before 1874 . In such a case sunspots in areas N21, S21 and L21 are omitted from cycle 21 (see Figure 2). As a compensation, however, sunspots from areas N22, S22, and L20 are included in cycle 21 although they actually belong to the next and the previous solar cycles. Since the number of those sunspots that are not compensated or that are overcompensated is very small (smaller than 1\%) compared to the total sunspot number (the so called intensity) of one cycle, it makes only little difference for the intensity to use the more correct sunspot numbers defined by the Maunder butterfly diagram rather than the standard definition. 
However, the standard definition may distort the calculated cycle lengths in a case when the two neighboring cycles are of significantly different amplitude. Figure 3 shows how the disk averaged sunspot number is formed from old and new sunspots in a case of equally high cycles (Figure 3(a)) and very different cycles (Figure 3(b)). In Figure 3(a) we have repeated the solar cycle 21 several times with a 11-year shift. Although the duration of the full cycle is 15 years, sunspot numbers depict the 11-year cycle length by construction. In Figure 3(b) we have reduced the amplitude of the second cycle by a factor of 5 keeping its time profile unchanged. (This roughly corresponds to the situation in the beginning of the Dalton minimum when a normally high solar cycle was followed by a minimum-like small cycle.) Although the cycles in Figure 3(b) still have the intrinsic 15-year full length and the 11-periodicity by construction, the standard cycle length calculation between total sunspot number minima yields 13 and 8 years for the high and low cycle, respectively. We note that other definitions of cycle length (using cycle maxima or medians as discussed below) would yield 11-year cycle lengths for both cycles.

As to the cycle length, it was shown by Mursula and Ulich (1998) that the cycle length as conventionally defined by the time interval between subsequent minima (min-min) or maxima (max-max) is very uncertain because of random fluctuations with uncertainties extending up to more than a year. Instead these authors suggested to define the cycle length between the median times (when half of the total SN over the cycle is reached) which are much more stable. In fact, cycle lengths defined from median times are accurate up to an uncertainty of a few days only. The sunspot cycle lengths as defined by the three different methods using the group sunspot number are shown in Table I.

\subsection{CyClicities IN SOlAR ACTIVITY}

The idea of regular variations in sunspot numbers was first suggested by the Danish astronomer Christian Horrebow in 1770's on the basis of his sunspot observations in 1761-1769 (Gleissberg, 1952; Vitinsky, 1965). Unfortunately, his results were forgotten and the data lost. Later, in 1843, the amateur astronomer Schwabe established that the sunspot activity varies cyclically with the period of about 10 years. This was the beginning of the study of cyclic variations of solar activity. In this work we are primarily interested in time scales of the order of the Schwabe cycle and longer.

The most prominent cycle in the sunspot series and in all solar activity is the 11year Schwabe cycle. This cyclicity is a fundamental feature of solar activity. The 11-year cyclicity is known in many other solar, heliospheric, geomagnetic, space weather, climate, etc. parameters.

The long-term change (trend) in the Schwabe cycle amplitude is known as the secular Gleissberg cycle (Gleissberg, 1944). However, the Gleissberg cycle is not a cycle in the strict periodic sense but rather a modulation of the cycle envelope with a varying time scale of 60-120 years (e.g., Gleissberg, 1971; Kuklin, 1976). On 
TABLE I

Dates of extremum and median times and lengths of group sunspot cycles as defined using minima (Min), maxima (Max) and medians (Med).

\begin{tabular}{|c|c|c|c|c|c|c|}
\hline \multirow[t]{2}{*}{ Cycle No. } & \multicolumn{3}{|c|}{ Date } & \multicolumn{3}{|c|}{ Length } \\
\hline & Min & Max & Med & Min-Min & Max-Max & Med-Med \\
\hline-4 & 1700 & 1705.4 & 1706 & 12.3 & 14.8 & 13.7 \\
\hline-3 & 1712.3 & 1720.2 & 1719.7 & 11.4 & 8.8 & 8.8 \\
\hline-2 & 1723.7 & 1729 & 1728.5 & 10 & 9.1 & 9.8 \\
\hline-1 & 1733.7 & 1738.1 & 1738.3 & 10.2 & 11.1 & 10.9 \\
\hline 0 & 1743.9 & 1749.2 & 1749.2 & 11.6 & 12.3 & 11.75 \\
\hline 1 & 1755.5 & 1761.5 & 1760.95 & 10.5 & 8.2 & 9.55 \\
\hline 2 & 1766 & 1769.7 & 1770.5 & 9.6 & 8.7 & 9.3 \\
\hline 3 & 1775.6 & 1778.4 & 1779.8 & 8.7 & 9.7 & 9.7 \\
\hline 4 & 1784.3 & 1788.1 & 1789.5 & 14 & 17.1 & 13.35 \\
\hline 5 & 1798.3 & 1805.2 & 1802.85 & 12.3 & 11.2 & 14.35 \\
\hline 6 & 1810.6 & 1816.4 & 1817.2 & 12.7 & 13.5 & 12.05 \\
\hline 7 & 1823.3 & 1829.9 & 1829.25 & 10.6 & 7.4 & 8.65 \\
\hline 8 & 1833.9 & 1837.3 & 1837.9 & 9.6 & 10.8 & 11.5 \\
\hline 9 & 1843.5 & 1848.1 & 1849.4 & 12.5 & 12 & 11.65 \\
\hline 10 & 1856 & 1860.1 & 1861.05 & 11.2 & 10.5 & 10.45 \\
\hline 11 & 1867.2 & 1870.6 & 1871.5 & 11.7 & 13.3 & 12.15 \\
\hline 12 & 1878.9 & 1883.9 & 1883.65 & 10.7 & 10.2 & 10.65 \\
\hline 13 & 1889.6 & 1894.1 & 1894.3 & 12.1 & 12.9 & 12.65 \\
\hline 14 & 1901.7 & 1907 & 1906.95 & 11.9 & 10.6 & 11.1 \\
\hline 15 & 1913.6 & 1917.6 & 1918.05 & 10 & 10.8 & 9.95 \\
\hline 16 & 1923.6 & 1928.4 & 1928 & 10.2 & 9 & 10.45 \\
\hline 17 & 1933.8 & 1937.4 & 1938.45 & 10.4 & 10.1 & 10.05 \\
\hline 18 & 1944.2 & 1947.5 & 1948.5 & 10.1 & 10.4 & 10.05 \\
\hline 19 & 1954.3 & 1957.9 & 1958.55 & 10.6 & 11 & 11.3 \\
\hline 20 & 1964.9 & 1968.9 & 1969.85 & 11.6 & 11 & 10.95 \\
\hline 21 & 1976.5 & 1979.9 & 1980.8 & 10.3 & 9.7 & 9.9 \\
\hline 22 & 1986.8 & 1989.6 & 1990.7 & 9.9 & 10.9 & 10.3 \\
\hline 23 & 1996.7 & 200.5 & 2001 & & & \\
\hline
\end{tabular}



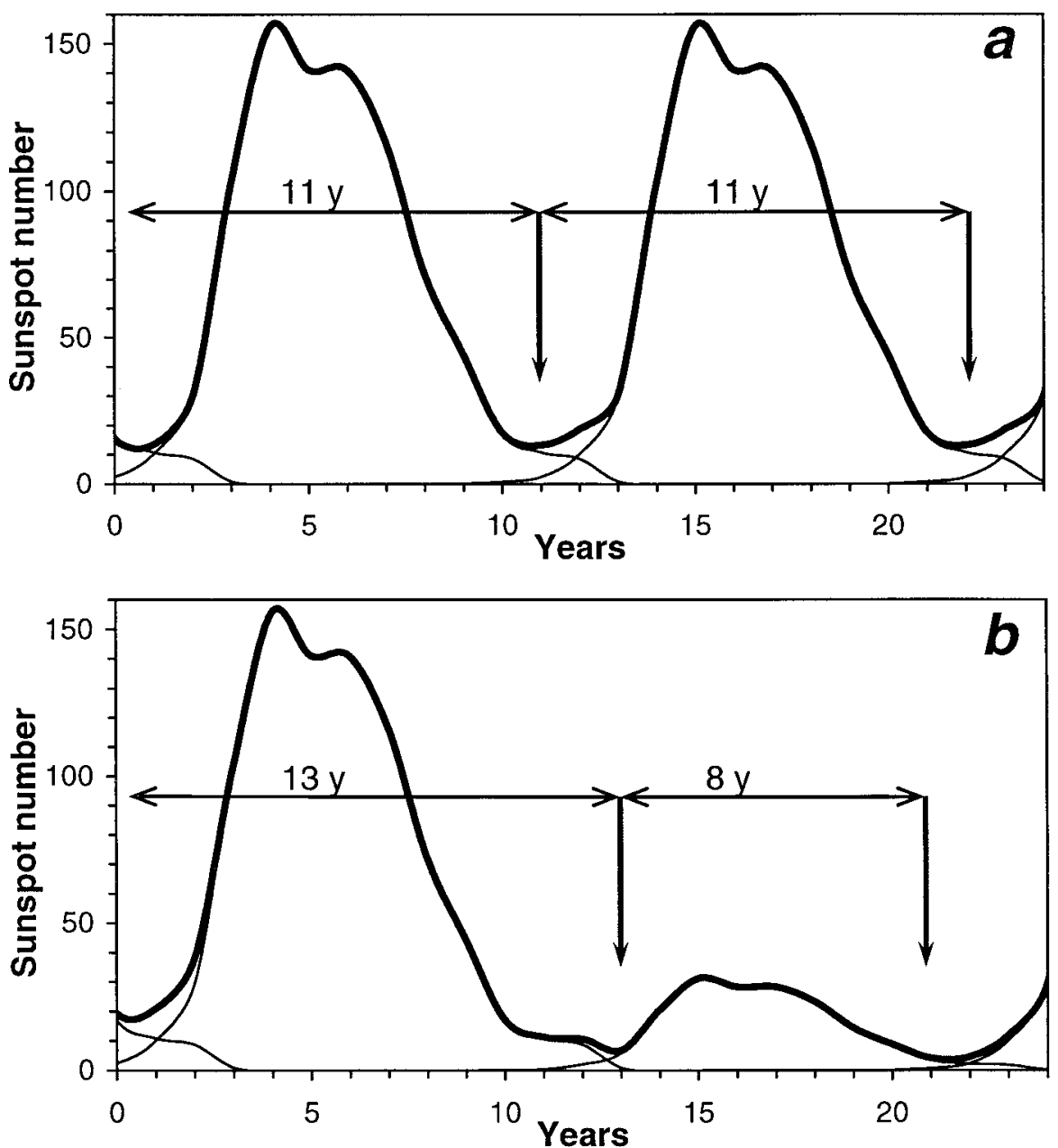

Figure 3. Sunspot numbers shown separately for the old and new cycles (thin lines) and integrated over the disk (thick solid curve). The cycle shape corresponds to cycle 21. Cycle shape is repeated several times with the 11-year time shift. Vertical arrows denote the time of sunspot number minimum. (a) Cycles with equal amplitude. (b) Second cycle has been reduced by a factor 5 .

the other hand, it is quite stable on long time scales (Feynman and Fougere, 1984; Feynman and Gabriel, 1990). In simple models (see, e.g., Sonett, 1983), sunspot activity is considered as an 11-year sinusoid which is amplitude modulated by the secular cycle. Even longer (super-secular) cycles are found in cosmogenic isotope data. Most prominent are the 205-210-year De Vries (Suess) cycle, a 600-700year cycle and a 2000-2400-year cycle (see, e.g., Vitinsky, Kopecký, and Kuklin, 1986, and references therein).

The background for the 11-year Schwabe cycle is the 22-year Hale magnetic polarity cycle. Hale found that the polarity of sunspot magnetic field changes in both 
hemispheres when a new 11-year cycle starts (Hale, 1908). This relates to the reversal of the global magnetic field of the Sun with the period of 22 years. It is often considered that the 11-year Schwabe cycle is the modulus of the sign-alternating Hale cycle (e.g., Sonett, 1983; Bracewell, 1986; Kurths and Ruzmaikin, 1990; De Meyer, 1998; Mininni, Gomez, and Mindlin, 2001). A hierarchy of sunspot cycles was presented, e.g., by Mordvinov and Kuklin (1999).

\subsection{WALDMEIER RELATIONS}

The 11-year solar cycle has an asymmetric shape with a shorter ascending $(\approx 4$ year on an average) and a longer $(\approx 7$-year) descending phase. The asymmetry is larger for shorter cycles, but the shape of individual cycles may vary. The cycle length varies from 7.5 to about 17 years in the GSN series (see Table I), the longest being the max-max length of cycle 4 . The amplitude of cycles also changes greatly, up to 200 in monthly sunspot numbers. The so called Waldmeier relations relate the amplitude and the duration of different phases of a solar cycle as follows (Waldmeier, 1935): (i) there is a strong negative correlation (the cross-correlation coefficient is $r=-0.83$ including cycles up to 22nd) between the duration of the ascending phase of a cycle and its amplitude; (ii) the relation between the duration of the descending phase and the cycle amplitude is weakly positive $(r=0.41)$. Together (i) and (ii) yield a weak negative relation between the amplitude and length of the solar cycle with the corresponding cross-correlation ranging from -0.3 to -0.5 depending on the cycle length definition (see Section 3.1). However, the negative relation is quite strong $(r=-0.65)$ between the amplitude of one cycle and the length of the preceding cycle (e.g., Solanki et al., 2002) which is expected from the dynamo action (e.g., Charbonneau and Dikpati, 2000). Surprisingly, a significant negative correlation $(r=-0.6)$ between the $i$ th cycle amplitude and $(i-3)$ th cycle length was also found (Orfila et al., 2002; Solanki et al., 2002), but the latter relation is valid only after the Dalton minimum.

\subsection{GREAT MINIMA AND PHASE CATASTROPHES OF SOLAR ACTIVITY}

Sometimes the regular time evolution of solar activity is intervened by periods of greatly depressed activity called great minima. The last great minimum (and the only one covered by direct solar observations) was the famous Maunder minimum during 1645-1715 (Eddy, 1976, 1983). Other great minima in the past known from cosmogenic isotope data include Spörer minimum in about 1450-1550, Wolf minimum around 12th century, etc. Great minima are an enigma for the solar dynamo theory. It is intensely debated what is the mode of the solar dynamo during such periods and what causes such minima (e.g., Feynman and Gabriel, 1990; Sokoloff and Nesme-Ribes, 1994; Schmitt, Schüssler, and Ferriz-Mas, 1996). Sometimes the Dalton minimum in about $1790-1820$ is also considered as a great minimum. However, sunspot activity was not completely suppressed and still showed the Schwabe cyclicity during the Dalton minimum. As suggested, e.g., by Schüssler, Schmitt, 

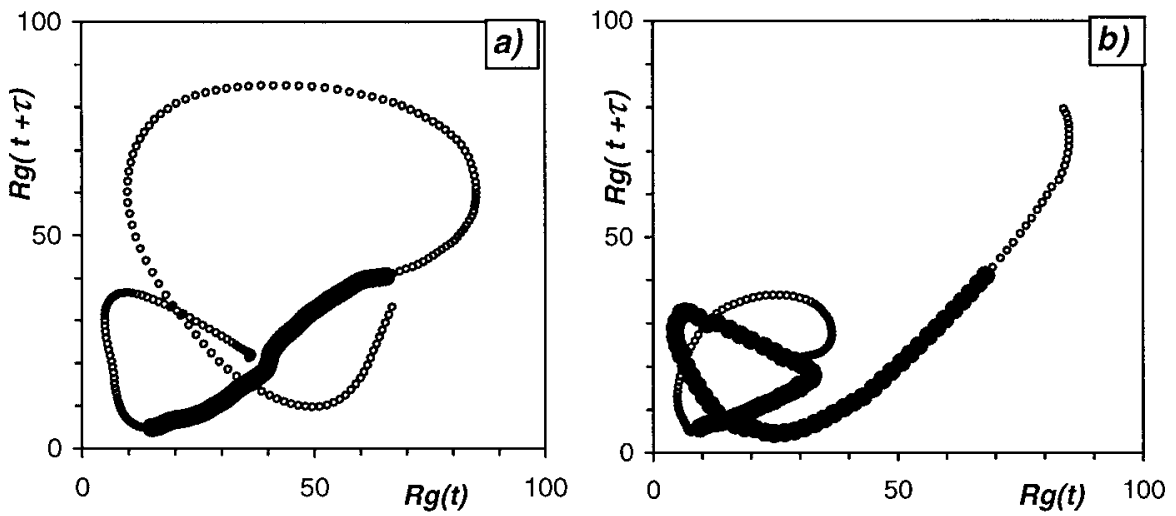

Figure 4. 2D projection of the phase evolution of sunspot activity around the Dalton minimum using the group sunspot numbers. Black dots denote the phase catastrophe in 1790-1798. (a) The standard sunspot number evolution depicting a phase catastrophe. (b) The same period but using the newly suggested lost cycle in 1793-1800 which removes the phase catastrophe.

and Ferriz-Mas, (1997) this can be a separate, intermediate state of the dynamo between the great minimum and normal activity. The Dalton minimum is often connected to the so-called phase catastrophe of solar activity evolution (see, e.g., Vitinsky, Kopecký, and Kuklin, 1986; Kremliovsky, 1994) which occurred in the beginning of the Dalton minimum. The phase catastrophe is shown in Figure 4(a) using a $2 \mathrm{D}$ projection (the method of time delayed components) of the sunspot activity phase evolution, similar to Figure 2 of Kremliovsky (1994), Figure 38 of Vitinsky, Kopecký, and Kuklin (1986), and Figure 5 of Serre and Nesme-Ribes (2000).

The phase catastrophe (1790-1798) is the period when the solar cycle evolution was not cyclic but roughly linear with greatly reduced phase evolution rate along the trajectory. A peculiarity in the phase evolution of sunspot activity around 1800 was also noted by Sonett (1983) who ascribed it to a possible error in Wolf data and by Wilson (1988) who reported on a possible misplacement of SN minima for cycles 4-6 in the WSN series.

Another period associated with phase catastrophe is the period of sunspot activity recovery after the Maunder minimum. The recovery of the 11-year cycle passed through the time of distorted phase evolution (Usoskin, Mursula, and Kovaltsov, 2001a) with the concurrent lost of the 88-year cycle phase (Feynman and Gabriel, 1990).

\subsection{RANDOMNESS IN SOLAR ACTIVITY}

The sunspot number series contains some noise which is larger than the observational uncertainties and this noise is thus a part of the real data. It is important to note that this noise is not white but rather 'colored' or correlated noise (e.g., Ostryakov and Usoskin, 1990b; Oliver and Ballester, 1996; Frick et al., 1997), 
i.e., the variance of noise depends on the level of sunspot activity. However, after normalization of the noise to the current average level of sunspot activity, the distribution of such dimensionless noise is nearly Gaussian, implying the existence of random fluctuations (Usoskin, Mursula, and Kovaltsov, 2001b).

Earlier it was common to describe sunspot activity as a multi-harmonic process with several basic harmonics (e.g., Vitinsky, 1965; Sonett, 1983; Vitinsky, Kopecký, and Kuklin, 1986, and references therein). Fluctuations of the observed sunspot numbers were believed to be due to noise which is added to the regular part and plays no role in the solar cycle evolution. This approach is oversimplified, depends on the chosen reference time interval and does not adequately describe the long-term evolution (see, e.g., Rozelot, 1994). The fact that purely mathematical/statistical models cannot give good predictions of solar activity (as will be discussed later) implies that the nature of solar cycle is not a multi-periodic or other purely deterministic process, but random (chaotic or stochastic) processes play an essential role in sunspot formation.

A new concept of the solar cycle as a low-dimensional chaotic system was introduced by Ruzmaikin (1981), and since early 1990's, many authors have considered solar activity as an example of low-dimensional deterministic chaos, described by the so called strange attractor (e.g., Kurths and Ruzmaikin, 1990; Ostryakov and Usoskin, 1990a; Morfill, Scheingraber, and Voges, 1991; Mundt and Maguire, 1991; Rozelot, 1995; Salakhutdinova, 1999; Serre and Nesme-Ribes, 2000). Randomness is a natural factor in the time series realization of such processes. However, parameters of the low-dimensional attractor were different when obtained by different authors because the analyzed data set is too short (Carbonell, Oliver, and Ballester, 1993, 1994). Also, the results are dependent on the choice of filtering methods (Price, Prichard, and Hogenson, 1992). Developing this approach, Mininni, Gomez, and Mindlin $(2000,2001)$ suggested to consider sunspot activity as an example of a 2D Van der Pol relaxation oscillator with an intrinsic stochastic component.

Interesting results were obtained using the approach suggested by Ruzmaikin $(1997,1998)$. The theory of magnetic flux emergence predicts a threshold for the buoyancy of magnetic tubes which results in sunspot formation (see, e.g., Schüssler et al., 1994; Caligari, Schüssler, and Moreno-Insertis, 1998; Dikpati et al., 2002). The regular magnetic field generated by the dynamo in the bottom of the convection zone is below the threshold (see, e.g., Zeldovich, Ruzmaikin, and Sokoloff, 1983; Schüssler et al., 1994). Accordingly, Ruzmaikin (1997) suggested that a randomly fluctuating field plays an important role so that only a combination of the regular dynamo and random fields can exceed the buoyancy threshold. This phenomenological model qualitatively reproduces some features of the sunspot cycle. Developing the idea of Ruzmaikin, Usoskin, Mursula and Kovaltsov (2001b) built a simple model of sunspot activity which includes a superposition of a regular 11-year oscillating dynamo related field, a weak permanent (relic) magnetic field and a randomly fluctuating component. This simple model was found to reproduce 
fairly well most of the fundamental features of sunspot cyclicity both for normal times and great minima. Note that no other model described above discussed the sunspot cycle behaviour during great minima. The fact that the model (Usoskin, Mursula, and Kovaltsov, 2001b) reproduces sunspot activity in two very different conditions with the same parameters of the random and permanent components only changing the level of dynamo gives additional support for the idea of the relic field in the Sun (see Section 5).

Not only phenomenological or basic principles models were used to understand the nature of randomness in sunspot activity. Corresponding theoretical dynamo models have also been developed which include stochastic processes (e.g., Weiss, Cattaneo, and Jones, 1984; Feynman and Gabriel, 1990; Schmalz and Stix, 1991; Lawrence, Cadavid, and Ruzmaikin, 1995; Schmitt, Schüssler, and Ferriz-Mas, 1996; Charbonneau and Dikpati, 2000; Charbonneau, 2001). E.g., Feynman and Gabriel (1990) suggested that the transition from regular to the chaotic dynamo passes through bifurcation. Charbonneau and Dikpati (2000) studied stochastic fluctuations in a Babcock-Leighton dynamo model and succeeded to qualitatively reproduce the Waldmeier anti-correlation between cycle amplitude and length. Their model also predicts a phase-lock of the Schwabe cycle, i.e., that the 11year cycle is an internal 'clock' of the Sun. Note that a significant fluctuating component (with the amplitude more than $100 \%$ of the regular component) is essential in their model. Durney (2000) and Charbonneau (2001) demonstrated that a Leighton-Babcock dynamo can be reduced to a one-dimensional iterative return map. This return map naturally produces the Gnevyshev-Ohl rule (in the form of cycle amplitude alteration; see also later) but the phase of this rule is not locked and may occasionally suffer a random phase jump. Again, the presence of noise is essential in this model.

While the existence of regularity and randomness in sunspot series is obvious, their mutual relationship is not clear (see, e.g., Wilson, 1994). The regular component of SN dominates during the normal activity times, while the sunspot occurrence was seemingly sporadic during the Maunder minimum. Moreover, the question is still open if randomness in sunspot data is due to chaotic or stochastic processes.

\subsection{PREDICTABILITY OF SUNSPOT ACTIVITY}

Randomness in the SN series is directly related to the predictability of solar activity. Forecasting solar activity is a subject of intensive studies since long (e.g., Yule, 1927; Newton, 1928; Gleissberg, 1948; Vitinsky, 1965). All prediction methods can be classified as regression (statistical) or precursor techniques or their combinations (Hathaway, Wilson, and Reichmann, 1999). Methods of the first class, including also a low-dimensional solar attractor representation (Kurths and Ruzmaikin, 1990), are based solely on the statistical properties of sunspot activity and give reasonable results on short-term forecasting. However, their prediction ability of the long-term activity is quite poor (see, e.g., reviews by Conway, 1998; 
Hathaway, Wilson, and Reichmann, 1999; Kane, 2001; Li, Yun, and Gu, 2001). E.g., only few predictions ( 3 out of 37 analyzed in Li, Yun, and Gu, 2001) of the maximum sunspot number of cycle 22 appeared close (within 10\%) to the observed value of 157.6 (see Figure 5(a)). The situation is similar with the prediction of cycle 23: only 4 out of 37 predictions were close to the observed annual maximum sunspot number 119.6 .

The prediction methods of the second class are based on a physical relation between the poloidal solar magnetic field, estimated from geomagnetic activity in the declining phase of the preceding cycle or in the minimum time (e.g., Hathaway, Wilson, and Reichmann, 1999), with the toroidal field responsible for sunspot formation. They usually yield better predictions of a forthcoming cycle maximum than the statistical methods, e.g., 10 out of 26 precursor predictions analyzed by $\mathrm{Li}$, Yun, and $\mathrm{Gu}$ (2001) lie close to the observed value (Figure 5(b)).

It has been shown using, e.g., such characteristics of the SN time series as the Lyapunov exponent (Ostryakov and Usoskin, 1990a; Kremliovsky, 1995) or wavelet entropy (Sello, 2000) that the applicability of regression prediction methods does not exceed one solar cycle. This is related to a question if an internal 'memory' exists in the solar dynamo which is expected in some dynamo models (see, e.g., Ossendrijver, Hoyng, and Schmitt, 1996). If such a memory does exist, sunspot activity could be predicted at least at the time scale of the memory. E.g., Balthasar and Schüssler $(1983,1984)$ suggested, studying preferred longitudes of sunspot occurrence, that there is a memory of one-two cycles, but the presence of a memory in SN was not confirmed by Oliver and Ballester (1998). Berdyugina and Usoskin (2003) have shown that migrating active longitudes of sunspot formation are not related to the memory of the dynamo but to au intrinsic feature of the spatial distribution of new sunspots which is persistent throughout the last 130 years. On the other hand, recent results on a possible three-cycle relation within the SN time series (Orfila et al., 2002; Solanki et al., 2002) might imply that a kind of memory exists even on the time scale of several decades. E.g., using the three-cycle quasiperiodicity found in cosmic rays and in the geomagnetic Ap index, Ahluwalia (1998) successfully predicted the amplitude of the cycle 23.

\section{Maunder Minimum (1645-1700)}

The Maunder minimum was well covered (more than $95 \%$ of days) by direct sunspot observations (Hoyt and Schatten, 1996). On the other hand, sunspots appeared only rarely, during $\approx 2 \%$ of days, and seemingly sporadically, making standard time series analysis methods fail (e.g., Frick et al., 1997). In such a case when daily sunspot numbers are small and large uncertainties of individual observations are possible, the most reliable information is whether a sunspot has been reported on a given day or not. This means that reliable sunspot activity can be determined not by the number of spots on the solar disk but by the frequency of sunspot 

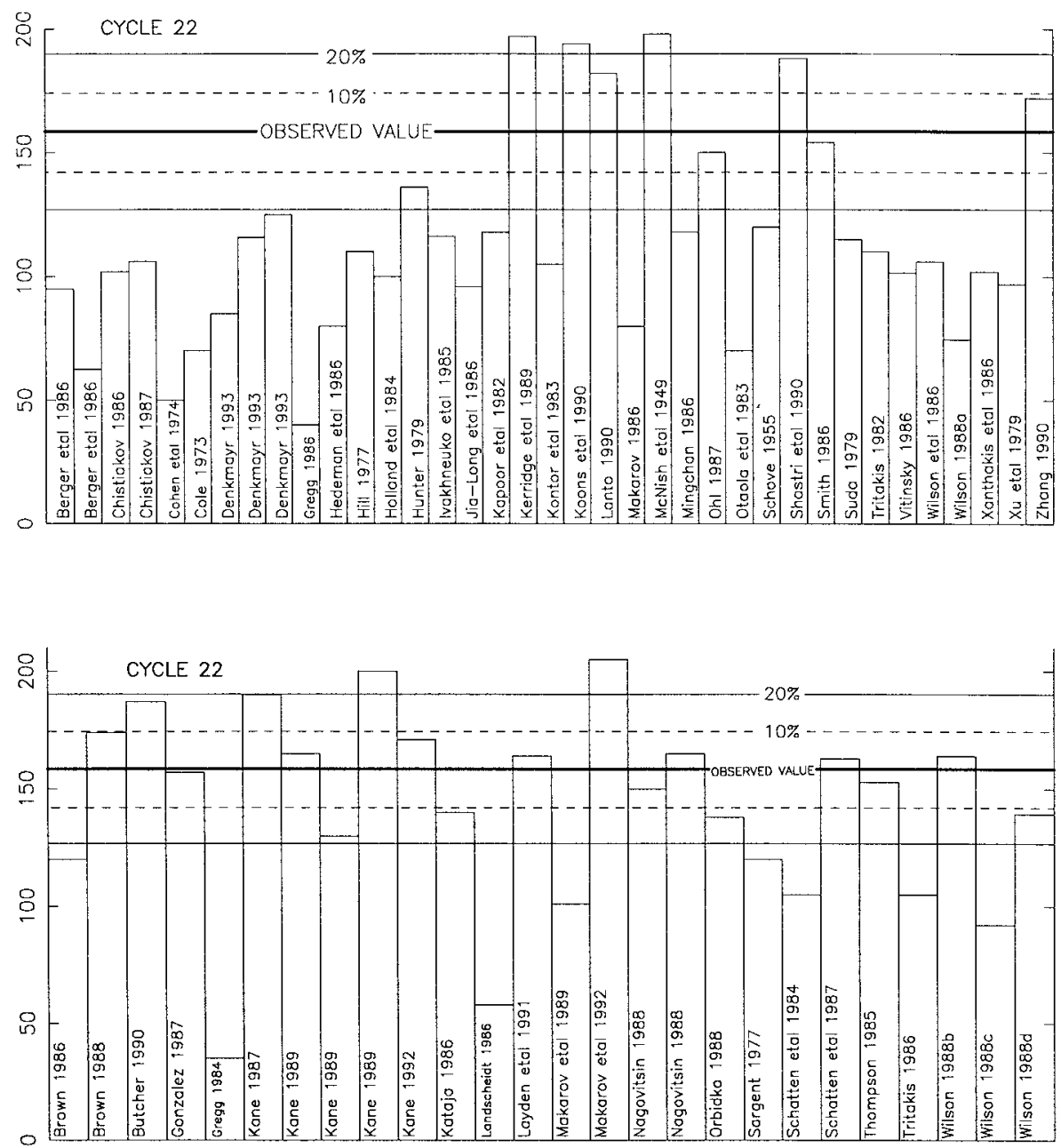

Figure 5. Predictions of the maximum sunspot number of solar cycle 22 (after Li, Yun, and Gu, 2001) using (a) mathematical/statistical methods based solely on sunspot activity features and (b) precursor methods.

occurrence. This approach was used to study the distribution of spotless days vs. days with sunspots around solar minima (e.g., Harvey and White, 1999). The occurrence of sunspot days during the Maunder minimum is shown in Figure 6(c). It is seemingly sporadic and without an indication of the 11-year cycle during the deep minimum (e.g., Letfus, 2000). Using a technique developed for the analysis of sparsely occurring events, Usoskin, Mursula, and Kovaltsov (2000, 2001a) demonstrated that the sunspot occurrence is gathered into two large clusters (shaded in Figure 6(a)) in 1652-1662 and 1672-1689 with the mass centers of these clusters in 1658 and 1679-1680 (Figure 6(b)). Together with the sunspot maxima before (1640) and after (1705) the deep Maunder minimum, this implies a dom- 


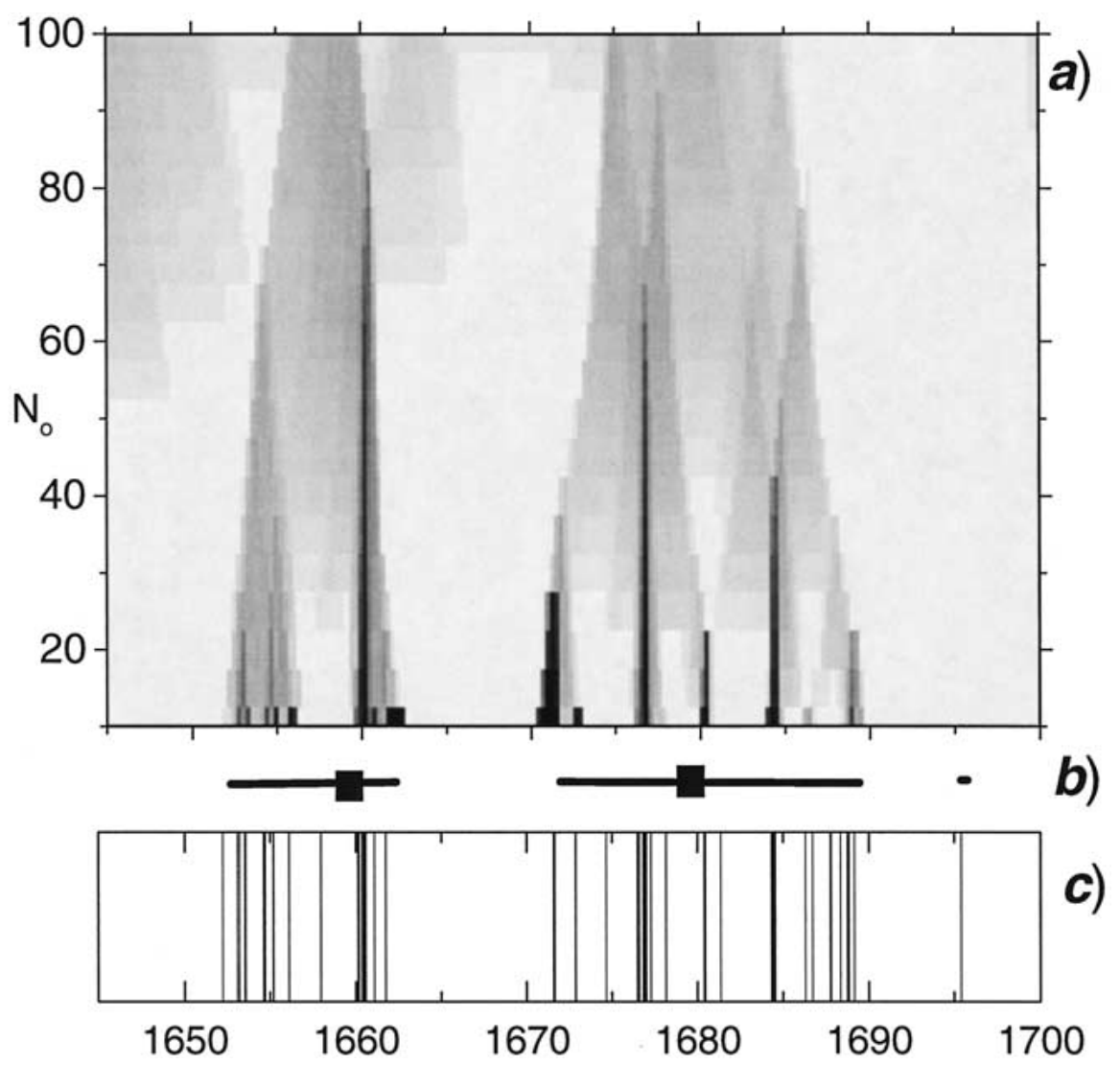

Figure 6. Occurrence of sunspot days during the Maunder minimum (after Usoskin, Mursula, and Kovaltsov, 2001a). (a) Dynamical map of sunspot day concentration for different scales. (b) Intervals of sunspot occurrence with the mass centers. (c) Sunspot days during the deep Maunder minimum.

inant 22-year periodicity in sunspot activity throughout the Maunder minimum. This dominant 22-year cyclicity also appear clearly as long spotless periods in 1645-1651, 1662-1671, and 1689-1695. A subdominant 11-year cycle emerges in SN towards the end of the Maunder minimum (Ribes and Nesme-Ribes, 1993; Mendoza, 1997; Usoskin, Mursula, and Kovaltsov, 2000) and becomes dominant again after 1700. This is in a general agreement with an earlier concept of an 'immersion' of 11-year cycles during the Maunder minimum (Vitinsky, Kopecký, and Kuklin, 1986, and references therein). This concept means that full cycles cannot be resolved and sunspot activity only appears as pulses around the cycle maximum times.

It is also interesting to note that sunspots were only seen in the southern solar hemisphere during the end of the Maunder minimum (Ribes and Nesme-Ribes, 1993; Sokoloff and Nesme-Ribes, 1994) which implies a significant asymmetry of Sun's surface magnetic field. 
The conclusion of the dominant 22-year cycle and a weak sub-dominant Schwabe cycle during the Maunder minimum (Usoskin, Mursula, and Kovaltsov, 2000, 2001 ) is in accordance with indirect solar proxy data: auroral occurrence (Křivský and Pejml, 1988; Schlamminger, 1990; Silverman, 1992) depicts a dominant 22year variability during that period, as well as the ${ }^{14} \mathrm{C}$ cosmogenic isotope concentration in tree rings (Kocharov et al., 1995; Peristykh and Damon, 1998). On the other hand, another cosmogenic isotope, the abundance of ${ }^{10} \mathrm{Be}$ in polar ice shows a dominant 11-year cycle during the Maunder minimum (Beer, Tobias, and Weiss, 1998). This may he, e.g., due to the effect of local climate on ${ }^{10} \mathrm{Be}$ precipitation (e.g., Lal, 1987; Beer et al., 1990; Steig et al., 1996). A detailed study is required to resolve this discrepancy.

The time behaviour of sunspot activity during the Maunder minimum yields the following general scenario (Vitinsky, Kopecký, and Kuklin, 1986; Ribes and Nesme-Ribes, 1993; Sokoloff and Nesme-Ribes, 1994; Usoskin, Mursula, and Kovaltsov, 2000, 2001a). Transition from the normal high activity to the deep minimum was sudden (within few years) without any apparent precursor. A 22-year cycle was dominant in SN occurrence during the deep minimum (1645-1700). The 11-year cycle was sub-dominant and became visible only towards the end of the minimum, starting to dominate the SN series after 1700. Recovery of sunspot activity from the deep minimum to normal activity was gradual passing through a period of nearly linear amplification of the 11-year cycle. It is interesting to note that such a qualitative evolution of a great minimum is predicted by the stochastically forced return map (Charbonneau, 2001) which is a truncation of the Babcock-Leighton dynamo model (see Figure 7): the onset of a great minimum occurs within one cycle (points 1355-1356 in Figure 7(a)) while the transition from the minimum to normal activity takes place through a gradual increase of cycle amplitudes (points 1313 onwards in Figure 7(b)). We note that a preliminary analysis of annual ${ }^{14} \mathrm{C}$ data during the Spörer minimum (the previous great minimum around 1450-1550) by Miyahara et al. (2003) reveals a similar pattern (constant 22-year cycle and suppressed 11-year cycle) supporting the above general scenario of great minima.

\section{22-Year Cycle in Sunspot Activity}

Although the 22-year magnetic cycle is the basis for the 11-year Schwabe cycle, a 22 -year cycle is not expected in the total (unsigned) $\mathrm{SN}$ if the dynamo process is symmetric with respect to the changing polarity. On the other hand, even a quick look at Figure 1 reveals a systematic alteration of cycle amplitudes. Gnevyshev and Ohl (1948) studied the intensity (total sum of SN over the cycle) of solar cycles and showed that solar cycles are coupled in pairs of a less intensive even-numbered cycle followed by a more intensive odd cycle. This is called the Gnevyshev-Ohl (G-O) rule which is much more stable than its simplified form based on the cycle amplitude alteration. Using WSN, the G-O rule works only since cycle 10 and fails 


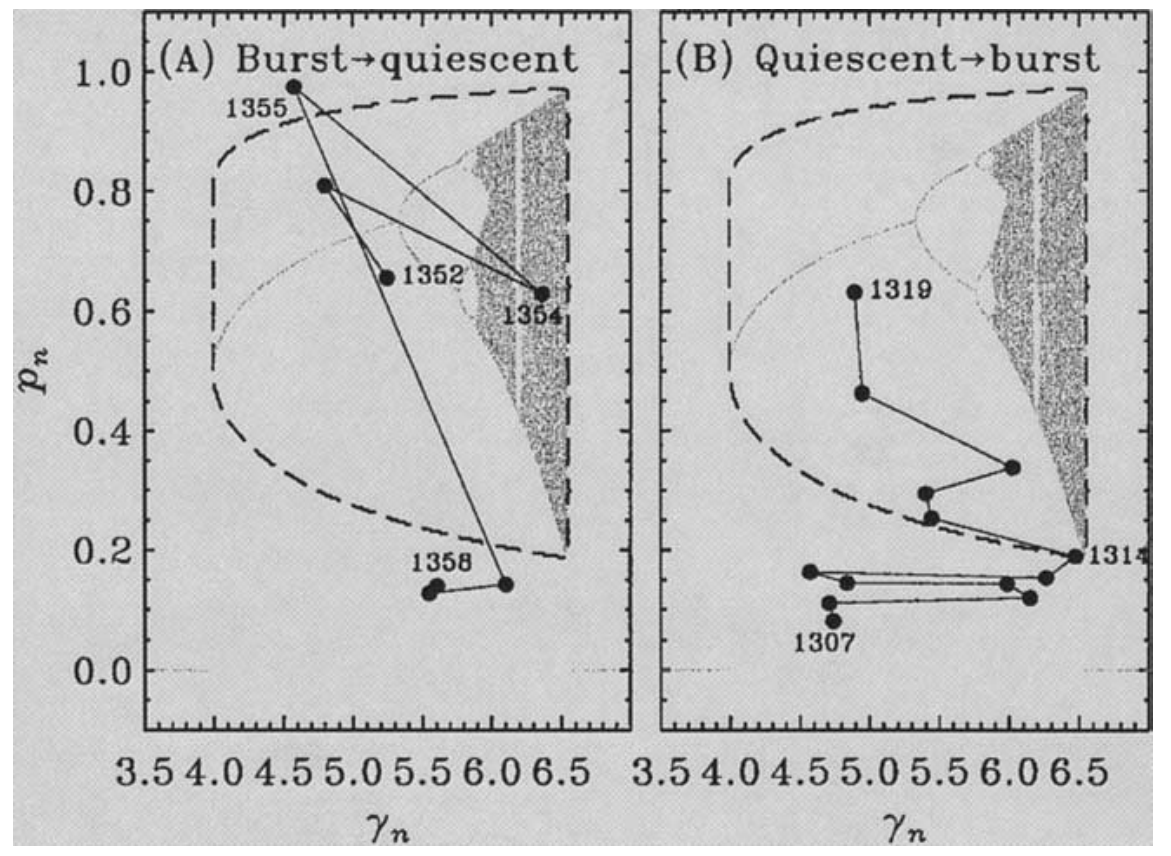

Figure 7. An onset (a) and offset (b) of a great minimum according to the stochastically forced return map simulations (after Charbonneau, 2001). Values of $p_{n}$ and $\gamma_{n}$ are related to the intensity and dynamo number of a solar cycle.
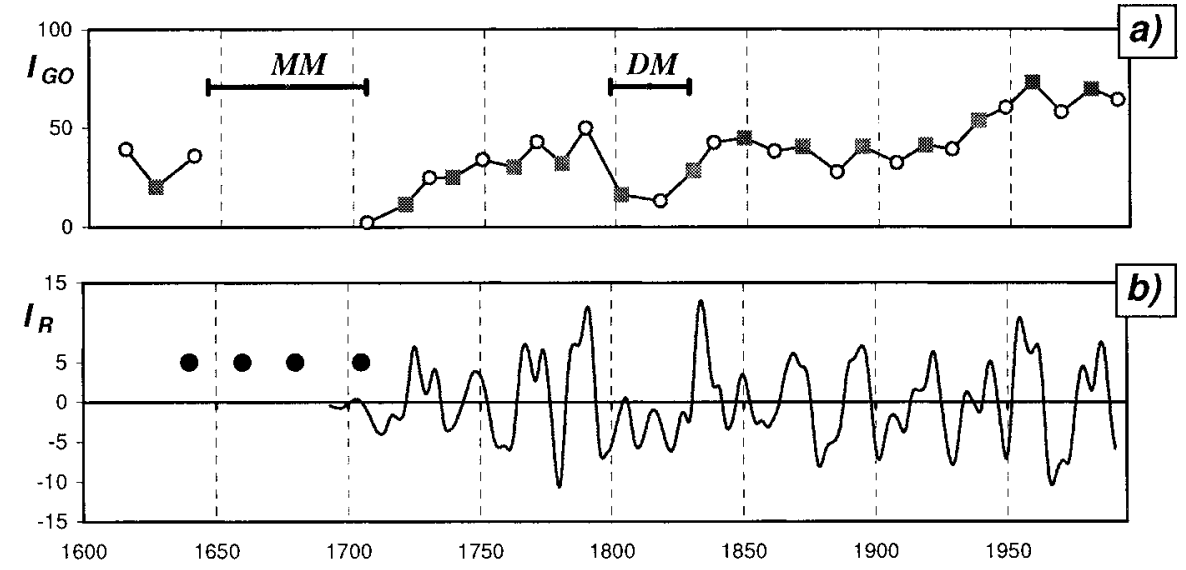

Figure 8. Illustration of the 22-year cycle in sunspot activity (after Mursula, Usoskin, and Kovaltsov, 2001). (a) Sunspot cycle intensities for odd (black squares) and even (open circles) sunspot cycles. (b) Band-pass filtered intensity of GSN series. The times of the 22-year cycle maxima before and during the Maunder minimum (Usoskin, Mursula, and Kovaltsov, 2000) are noted by black circles. 
for cycle pairs 4-5 and 8-9 (Gnevyshev and Ohl, 1948; Wilson, 1988; Storini and Sykora, 1997). When using GSN, the G-O rule is valid since the Dalton minimum (Figure 8) and, in the reverse order, even before that (Mursula, Usoskin, and Kovaltsov, 2001). It has been suggested that the $\mathrm{G}-\mathrm{O}$ rule will be broken for the ongoing cycle pair 22-23 (Komitov and Bonev, 2001). A phase jump of the $\mathrm{G}-\mathrm{O}$ rule is possible, e.g., in the framework of a reduced Leighton-Babcock dynamo model (Charbonneau, 2001). A careful analysis of the GSN series reveals (Mursula, Usoskin, and Kovaltsov, 2001) that there exists a persistent 22-year cyclicity in the sunspot data since the Maunder minimum (see Figure 8(b)). Taking also into account the dominant 22-year cyclicity in SN during the Maunder minimum (Usoskin, Mursula, and Kovaltsov, 2000) and the obvious cycle amplitude alteration before the Maunder minimum (both are shown by black dots in Figure 5(b)), one can see that the 22-year periodicity is present in sunspot activity throughout the entire interval of about 400 years of solar observations. During the Maunder minimum this 22-year activity was dominant over the Schwabe cycle, and its maxima before, during and after the Maunder minimum are well in phase (see Figure 5), implying that the 22-year periodicity is persistent and phase-locked. In particular there is no phase reversal across the Dalton minimum, contrary to the $\mathrm{G}-\mathrm{O}$ rule based on standard cycle numbering. The peak-to-peak amplitude of the 22-year cycle is roughly constant (see Figure 8(b)) and independent of the solar activity level, i.e., its amplitude does not correlate with the current level of solar activity. This 22-year cycle is the underlying pattern behind the $\mathrm{G}-\mathrm{O}$ rule.

This 22-year cycle with a stable phase and a constant amplitude independent on the solar activity level was interpreted as a systematically asymmetric oscillation of the magnetic field in the convection zone (Mursula, Usoskin, and Kovaltsov, 2001). Such an asymmetry can be naturally explained if one assumes a weak constant magnetic field in the bottom of the convection zone. A relic magnetic field can survive in the Sun due to the high conductivity in the solar interior (Cowling, 1945; Sonett, 1983). Some evidences favouring this idea have been presented earlier (Sonett, 1983; Bravo and Stewart, 1995; Boruta, 1996). Due to a strong amplification by the dynamo fluid motions in the convection zone, such a weak constant field can interact with the poloidal/toroidal dynamo field and play a role in the formation of a sunspot cycle (Levy and Boyer, 1982; Sonett, 1983; Boyer and Levy, 1984). This hypothesis explains (Mursula, Usoskin, and Kovaltsov, 2001; Usoskin, Mursula and Kovaltsov, 2001b) the main pattern of the 22-year cycle: persistent phase and constant amplitude during the normal activity times and its dominance during the Maunder minimum.

\section{Dalton Minimum and the Lost Cycle}

The Dalton minimum (DM) around 1800 was a period when SN was reduced to have cycle maximum values of about 30 . The beginning of the Dalton minimum 


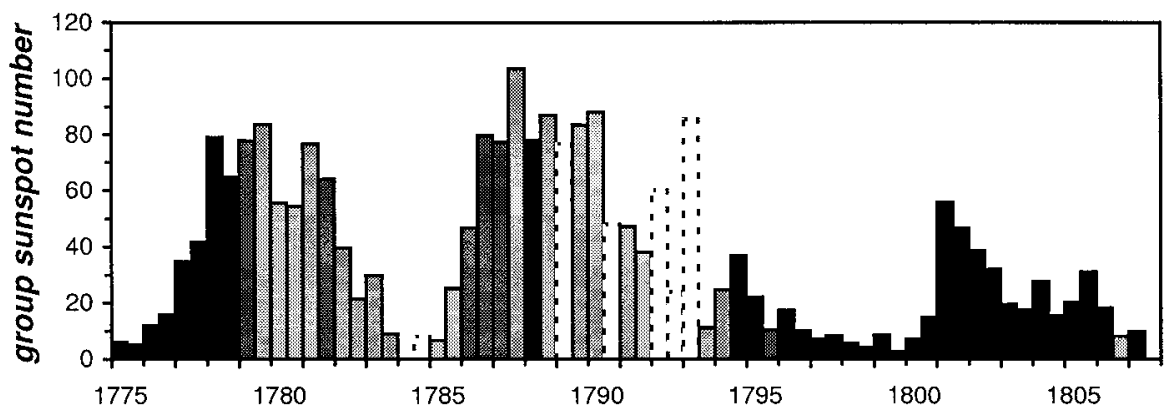

Figure 9. Semiannual GSN data at the beginning of Dalton minimum (after Usoskin, Mursula and Kovaltsov, 2002). White, light grey, dark grey and black shading denotes unreliable $(<6$ observation days during the corresponding 6 months), poorly reliable (6-12 days), reliable (13-24 days), and highly reliable ( $>24$ days) values.

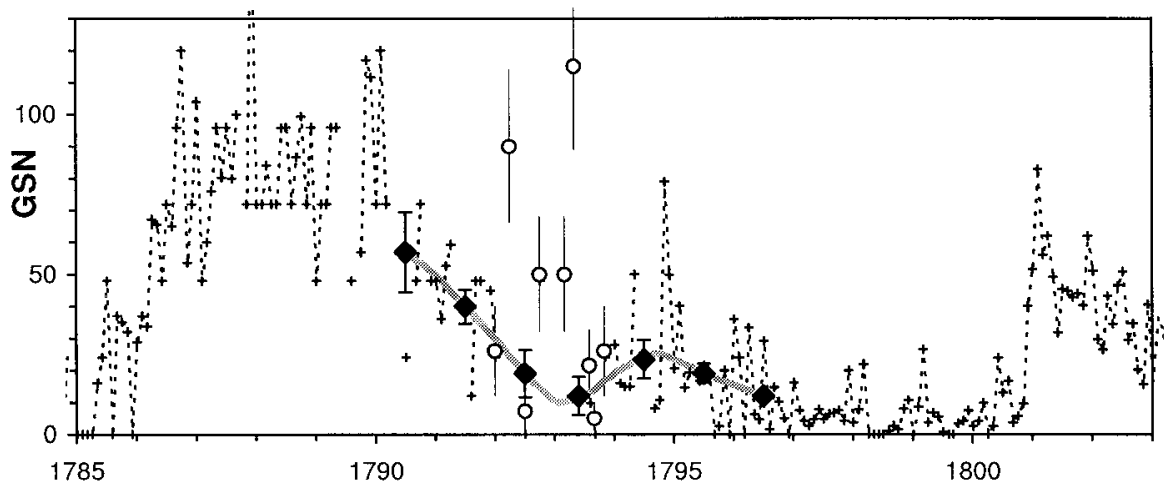

Figure 10. Group sunspot numbers circa 1800 (after Usoskin, Mursula and Kovaltsov, 2003), GSN values outside 1792-1793 are shown by the dashed curve, while open dots with error bars depict the estimated monthly means and their standard errors in 1792-1793. The solid diamonds present the estimated weighted annual averages in 1790-1796 with the spline fit to them.

was quite exceptional. The years $1790-1794$ were very poorly covered by sunspot observations (Figure 9), probably because of the unstable political situation in Europe after the French revolution in 1789. The WSN series was interpolated during those years leading to abnormally long cycle 4 (about 14, 17, and 14.5 years using min-min, max-max or median cycle length definitions). A strong anomaly in the cycle evolution during 1790's was found by many authors. E.g., Sonett (1983) suspected an error in the WSN series, and Wilson (1988) found that cycle minima were likely misplaced for cycles 4,5 and noted that 'clearly, something is amiss in Hale cycle 3'. Sello (2000) studied the wavelet entropy (a measure of disorder in the time series) of SN series. The absolute maximum of entropy falls onto the cycle 4 which implies high disorder in SN data during that time. It has earlier been suggested that this anomaly is related to a phase catastrophe of solar cycle evolution due to the very long ( $>10$ years) descending phase of cycle 4 when 

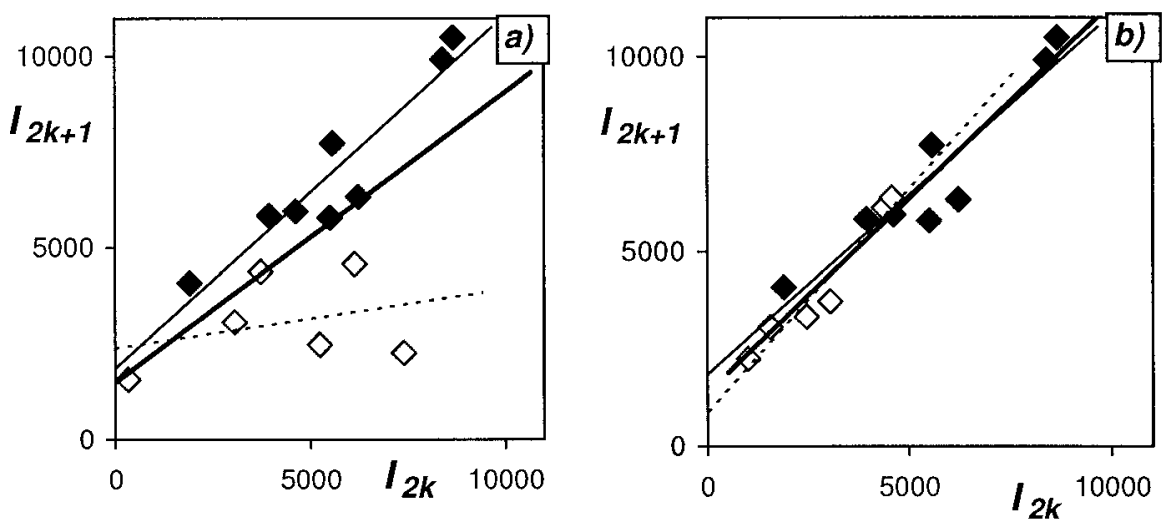

Figure 11. Illustration of the Gnevyshev-Ohl rule: intensities of odd sunspot cycles vs. even cycles (after Usoskin, Mursula, and Kovaltsov, 2001). (a) Standard cycle numbering. (b) Including the new cycle. Open (filled) diamonds correspond to the interval before (after) the Dalton minimum. Dotted, thin and thick solid lines give the linear correlation before and after the Dalton minimum, and for the entire period (1610-1996), respectively.

the SN evolution was not cyclic but linear (see Figure 4(a)), probably due to the linear interpolation over sparse data points (Vitinsky, Kopecký, and Kuklin; 1986; Kremliovsky, 1994). Moreover, the fact that the G-O rule suffered the phase reversal at this time suggests that the cycle numbering could be distorted circa 1800. Usoskin, Mursula, and Kovaltsov (2001b) suggested that one small cycle could have been lost in 1790's because of sparse and unreliable observations during that time. The new cycle (1793-1800) would be the first and smallest cycle of the Dalton minimum. The suggested time profile of SN during this interval is shown in Figure 10. This would remove all the problems related to the phase catastrophe, making the solar activity evolution cyclic (see also Figure 4(b)). It also makes the scenario of the Dalton minimum similar to that of the Maunder minimum: sudden descend of a normal high cycle to the lowest level without a precursor, followed by a gradual restoration of the activity level. The new cycle restores the order of the $\mathrm{G}-\mathrm{O}$ rule of cycle pairing making it valid now since 1610 (see Figure 11). Also, the new cycle does not distort the cycle length distribution or Waldmeier relations (Usoskin, Mursula, and Kovaltsov, 2002). The cycle definitions in this case are summarized in Table II (similar to Table I).

Unfortunately, the absence of latitudinal or magnetic polarity information of sunspots during the period under question makes it impossible to verify the suggested lost cycle directly, and one has to use indirect evidences. Usoskin, Mursula, and Kovaltsov (2002) demonstrated that, e.g., data on visual aurorae depict a peak in 1796 supporting the idea of the lost cycle. Also, direct measurements of the geomagnetic field declination yield a similar pattern, giving further support to the idea (Mursula, Usoskin, and Nevanlinna, 2003). The sensitivity of cosmogenic ${ }^{10} \mathrm{Be}$ and ${ }^{14} \mathrm{C}$ isotopes is not enough to resolve the small cycle (Usoskin, Mur- 
TABLE II

Dates of extremes and lengths of sunspot cycles (similar to Table I) around the Dalton minimum taking into account the lost cycle.

\begin{tabular}{llllllll}
\hline \multirow{2}{*}{ Cycle No. } & \multicolumn{3}{c}{ Date } & & \multicolumn{3}{c}{ Length } \\
\cline { 2 - 3 } \cline { 7 - 8 } \cline { 7 - 8 } & Min & Max & Med & & Min-Min & Max-Max & Med-Med \\
\hline $3^{\prime}$ & 1784.3 & 1788.4 & 1788.5 & & 8.7 & 6.4 & 6.7 \\
4 & 1793 & 1794.8 & 1795.2 & & 7 & 7.7 & 7.9 \\
5 & 1800 & 1802.5 & 1803.1 & & 10.8 & 14.6 & 14.1 \\
\hline
\end{tabular}

sula, and Kovaltsov, 2002). Recently Krivova, Solanki, and Beer (2002) called the existence of the new cycle in question claiming that the suggested new minimum in 1793 contradicts with SN statistics. However, as argued by Usoskin, Mursula, and Kovaltsov (2003a), the SN statistical features during 1792-1793 are typical for sunspot activity during the times of sunspot minima, thus favoring the additional minimum in 1793.

\section{Summary}

In this brief review we have discussed some recent achievements, mostly experimental and phenomenological, in the study of long-term solar cycle evolution. These new findings became possible, thanks to a tremendous work of Hoyt and Schatten (1998) who completed a series of group sunspot numbers with all basic information on observations available. The theoretical basis is in the recent developments of the dynamo and magnetic flux buoyancy theories. Below we list some interesting recent findings.

- Randomness is an essential intrinsic factor of the sunspot cycle although its nature is not yet completely understood. This implies a stochastic or chaotic component in sunspot activity which limits the predictability of the solar cycle. An essential random component is found necessary in recent theoretical models.

- The cyclic nature of sunspot activity during the Maunder minimum has been re-analyzed. A 22-year cycle was dominant during the deep Maunder minimum (1645-1700), and the 11-year cycle was gradually emerging towards the end of the minimum period.

- A persistent 22-year cycle with stable phase and roughly constant amplitude is present throughout the whole 400 -year long sunspot number series. The relation between the 11-year and the 22-year cycles (the latter was dominant during the Maunder minimum and sub-dominant during normal activity times) has been interpreted as a result of the mean field dynamo in the presence of a weak constant (relic) magnetic field in the Sun. 
- One small solar cycle may have been lost in 1790's because of extremely sparse sunspot observations. Introducing this new cycle removes the phase catastrophe in the beginning of the Dalton minimum and restores the order of the Gnevyshev-Ohl rule of cycle pairing throughout the 400-year long sunspot number series. Although the existence of this cycle cannot be confirmed directly, other independent, indirect evidence supports such an idea.

- A unified scenario of a great minimum has been suggested: a sudden suppression of sunspot activity to the lowest level (immersion of the cycles) followed by a gradual restoration of the activity level through the emergence of 11-year cycliaty. Such a behaviour is in agreement with some stochastically forced dynamo models.

\section{Acknowledgements}

The Academy of Finland is acknowledged for financial support. We thank Dr. G.A. Kovaltsov for fruitful discussions and the anonymous referee for useful comments.

\section{References}

Ahluwalla, H. S.: 1998, J. Geophys. Res. 103, 12103.

Balthasar, H. and Schüssler, M.: 1983, Solar Phys. 87, 23.

Beer, J., Tobias, S., and Weiss, N.: 1998, Solar Phys. 181, 237.

Beer, J. et al.: 1990, Nature 347, 164.

Berdyugina, S. V. and Usoskin, I. G.: 2003, Astron. Astrophys. 405, 1121.

Boruta, N.: 1996, Astrophys. J. 458, 832.

Boyer, D. W. and Levy, E. H.: 1984, Astrophys. J. 277, 848.

Bracewell, R. N.: 1986, Nature 323, 516.

Bravo S. and Stewart, G.: 1995, Astrophys. J. 446, 431.

Caligari, P., Schüssler, M., and Moreno-Insertis, F.: 1998, Astrophys. J. 502, 481.

Carbonell, M., Oliver, R., and Ballester, J. L.: 1993, Astron. Astrophys. 274, 497.

Carbonell, M., Oliver, R., and Ballester, J. L.: 1994, Astron. Astrophys. 290, 983.

Charbonneau, P.: 2001, Solar Phys. 199, 385.

Charbonneau, P. and Dikpati, M.: 2000, Astrophys. J. 543, 1027.

Conway, A. J.: 1998, New Astron. Rev. 42, 343.

Cowling, T. G.: 1945, Monthly Notices Royal Astron. Soc. 105, 167.

De Meyer, F.: 1998, Solar Phys. 181, 201.

Dikpati, M., Corbard, T., Thompson, M. J., and Gilman, P. A.: 2002, Astrophys. J. 575, L41.

Durney, B.: 2000, Solar Phys. 196, 421.

Eddy, J. A.: 1983, Solar Phys. 192, 1189.

Eddy, J. A.: 1976, Science 192, 1189.

Eddy, J. A.: 1983, Solar Phys. 89, 195.

Frick, P. et al.: 1997, Astron. Astrophys. 328, 670.

Feynman, J. and Fougere, P. F.: 1984, J. Geophys. Res. 89, 3023.

Feynman, J. and Gabriel, S. B.: 1990, Solar Phys. 127, 393.

Gleissberg, W. A.: 1944, Terrest. Magnetic. Atmospheric Electr. 49, 243.

Gleissberg, W. A.: 1948, Popular Astron. 56, 399.

Gleissberg, W. A.: 1952, Die Haufigkeit der Sonnenflecken, Akademie-Verlag, Berlin. 
Gleissber, W. A.: 1971, Solar Phys. 21, 240.

Gnevyshev, M. N. and Ohl, A. I.: 1948, Astron. Zh. 25, 18.

Hale, G. E.: 1908, Astrophys. J. 28, 315.

Harvey, K. L. and White, O. R.: 1999, J. Geophys. Res. 104, 19759.

Hathaway, D. H., Wilson, R. M., and Reichmann, E. J.: 1999, J. Geophys. Res. 104, 22375.

Hathaway, D. H., Wilson, R. M., and Reichmann, E. J.: 2002, Solar Phys. 211, 357.

Hoyt D. V. and Schatten, K.: 1996, Solar Phys. 165, 181.

Hoyt D. V. and Schatten, K.: 1998, Solar Phys. 179, 189.

Kane, R. P.: 2001, Solar Phys. 202, 395.

Kocharov, G. E. et al.: 1995, Solar Phys. 159, 381.

Komitov, B. and Bonev, B.: 2001, Astrophys. J. 554, L119.

Kremliovsky, M. N.: 1994, Solar Phys. 151, 351.

Kremliovsky, M. N.: 1995, Solar Phys. 159, 371.

Krivova, N. A., Solanki, S. K., and Beer, J.: 2002, Astron. Astrophys. 396, 235.

Křivský, L. and Pejml, K.: 1988, Astron. Inst. Czech. Acad. Sci. 75, 32.

Kuklin, G. V.: 1976, in Bumba and Kleczek (eds.), 'Basic Mechanisms of Solar Activity', Proc. 70th IAU Symp. 147.

Kurths, J. and Ruzmaikin, A. A. Ruzmaikin: 1990, Solar Phys. 126, 407.

Lal, D.: 1987, Geophys. Res. Lett. 14, 785.

Lawrence, J. K., Cadavid, A. C., and Ruzmaikin, A. A.: 1995, Astrophys. J. 455, 366.

Letfus, V.: 1999, Solar Phys. 184, 201.

Letfus, V.: 2000, Solar Phys. 194, 175.

Levy, E. H. and Boyer, D.: 1982, Astrophys. J. 254, L19.

Li, K. J., Yun, H. S., and Gu, X. M.: 2001, Astron. Astrophys. 368, 285.

Li, K. J., Yun, H. S., Liang, H. F., and Gu, X. M.: 2002, J. Geophys. Res. 107.

Mendoza, B.: 1997, Ann. Geophys. 15, 397.

Mininni, P. D., Gomez, D. O., and Mindlin, G. B.: 2000, Phys. Rev. Lett. 85, 5476.

Mininni, P. D., Gomez, D. O., and Mindlin, G. B.: 2001, Solar Phys. 201, 203.

Miyahara, H. et al.: 2003, in: Proc. 28th Int. Cosmic Ray Conf., Tsukuba 7, 4139.

Mordvinov, X. V. and Kuklin, G. V.: 1999, Solar Phys. 187, 223.

Morfill, G. E., Scheingraber, H. and Voges, W.: 1991, in: C. P. Sonnet, M. S. Giampapa, and M. S. Matthews (eds.), The Sun in Time, University of Arizona Press, Tucson, p. 30.

Mundt, M. D., Maguire, W. B. II, and Chase, R. R. P.: 1991, J. Geophys. Res. 96, 1705.

Mursula, K., Usoskin, I. G., and Kovaltsov, G. A.: 2001, Solar Phys. 198, 51.

Mursula, K., Usoskin, I. G., and Nevanlinna, H.: 2003, in preparation.

Mursula, K. and Ulich, Th.: 1998, Geophys. Res. Lett. 25, 1837.

Nagovitsyn, Yu. A.: 1997, Astron. Lett. 23, 74.

Newton, H. W.: 1928, Quart. J.R. Meteoral. Soc. 54, 161.

Oliver, R. and Ballester, J. L.: 1996, Solar Phys. 169, 215.

Oliver, R. and Ballester, J. L.: 1998, Phys. Rev. E. 58, 5650.

Orfila, A., Ballester, J. L., Oliver, R., Alvarez, A., and Tintoré, J.: 2002, Astron. Astrophys. 386, 313.

Ossendrijver, A. J. H., Hoyng, P., and Schmitt, D.: 1996, Astron. Astrophys. 313, 938.

Ostryakov, V. M. and Usoskin, I. G.: 1990a, Solar Phys. 127, 405.

Ostryakov, V. M. and Usoskin, I. G.: 1990b, Soviet. Tech. Phys. Lett. 16, 658.

Pelt, J., Brooke, J., Pulkkinen, P. J., and Tuominen, I.: 2000, Astron. Astrophys. 362, 1143.

Peristykh, A. N. and Damon, P. E.: 1998, Solar Phys. 177, 343.

Price, C. P., Prichard, D., and Hogenson, E. A.: 1992, J. Geophys. Res. 97, 19113.

Ribes, J. C. and Nesme-Ribes, E.: 1993, Astron. Astrophys. 276, 549.

Rigozo, N. R., Echer, E., Vieira, L. E. A., and Nordemann, D. J. R.: 2001, Solar Phys. 203, 179.

Rozelot, J. P.: 1994, Solar Phys. 149, 149.

Rozelot, J. P.: 1995, Astron. Astrophys. 297, L45. 
Ruzmaikin, A. A.: 1981, Comm. Modern Phys. C9, 85.

Ruzmaikin, A.: 1997, Astron. Astrophys. 319, L13.

Ruzmaikin, A.: 1998, Solar Phys. 181, 1.

Salakhutdinova, I. I.: 1999, Solar Phys. 188, 377.

Schlammmger, L.: 1990, Monthly Notices Royal Astron. Soc. 247, 67.

Schmalz, S. and Stix, M.: 1991, Astron. Astrophys. 245, 654.

Schmitt D., Schüssler, M., and Ferriz-Mas, A.: 1996, Astron. Astrophys. 311, L1.

Schove, D. J.: 1955, J. Geophys. Res. 60, 127.

Schröder, W.: 1992, J. Geomagn. Geoelectr. 44, 119.

Schüssler, M. P., Schmitt, D., and Ferriz-Mas, A.: 1997, in Advances in the Physics of Sunspots, B. Schmieder, J. C. del Toro Iniesta and M. Vazquez (eds.) A.S.P. Conf. Ser. 18, 39.

Schüssler, M. P., Caligari, P., Ferriz-Mas, A., and Moreno-Insertis, F.: 1994, Astron. Astrophys. 281, L69.

Sello, S.: 2000, Astron. Astrophys. 363, 311.

Serre, T. and Nesme-Ribes, E.: 2000, Astron. Astrophys. 360, 319.

Silverman, S. M.: 1983, J. Geophys. Res. 88, 8123.

Silverman, S. M.: 1992, Rev. Geophys. 30, 333.

Silverman, S. M.: 1998, J. Atmospheric Solar-Terrest. Phys. 60, 997.

Sokoloff, D. and Nesme-Ribes, E.: 1994, Astron. Astrophys. 288, 293.

Solanki, S. K., Krivova, N. A., Schüssler, M., and Fligge, M.: 2002, Astron. Astrophys. 396, 1029.

Sonett, C. P.: 1983, J. Geophys. Res. 88, 3225.

Steig, E. J. et al.: 1996, Geophys. Res. Lett. 23, 523.

Storini M. and Sykora, J.: 1997, Solar Phys. 176, 417.

Stuiver, M., and Quay, P.: 1980, Science 207, 11.

Usoskin, I. G., Mursula, K., and Kovaltsov, G. A.: 2000, Astron. Astrophys. 354, L33.

Usoskin, I. G., Mursula, K., and Kovaltsov, G. A.: 2001a, J. Geophys. Res. 106, 16039.

Usoskin, I. G., Mursula, K., and Kovaltsov, G. A.: 2001b, Solar Phys. 199, 187.

Usoskin, I. G., Mursula, K., and Kovaltsov, G. A.: 2002, Geophys. Res. Lett. 29.

Usoskin, I. G., Mursula, K., and Kovaltsov, G. A.: 2003a, Astron. Astrophys. 403, 743.

Usoskin, I. G., Mursula, K., and Kovaltsov, G. A.: 2003b, Solar Phys., in press.

Vitinsky, Yu. I.: 1965, Solar Activity Forecasting, Israel Program for Scientific Translations, Jerusalem.

Vitinsky, Yu. I., Kopecký, M., and Kuklin, G. V.: 1986, Statistics of Sunspot Activity, Nauka, Moscow. Waldmeier, M.: 1935, Astron. Mitt. Zürich 14, 105.

Waldmeier, M.: 1961, The Sunspot Activity in the Years 1610-1960, Zurich Schulthess and Company AG, Zürich.

Weiss, N. O., Cattaneo, F., and Jones, C. A.: 1984, Geophys. Astrophys. Fluid Dynamics 30, 305.

Wilson, P. R.: 1994, Solar and Stellar Activity Cycles, Cambridge University Press, Cambridge, $274 \mathrm{pp}$.

Wilson, R. M.: 1988, Solar Phys. 117, 269.

Wilson, R. M.: 1998, SoLar Phys. 182, 217.

Wittmann, A. D. and Xu, Z. T.: 1987, Astron. Astrophys. Suppl. Ser. 70, 83.

Yua, K. K. and Stephenson, F. R.: 1988, Quart. J.R. Astron. Soc. 29, 175.

Yule, G. U.: 1927, Phil. Trans. R. Soc. London A226, 267.

Zeldovich, Ya. B., Ruzmaikin, A. A., and Sokoloff, D. D., 1983: Magnetic Fields in Astrophysics, Gordon and Breach, New York. 